\title{
Nonreciprocal Gain in Non-Hermitian Time-Floquet Systems
}

\author{
Theodoros T. Koutserimpas and Romain Fleury ${ }^{*}$ \\ Laboratory of Wave Engineering, Ecole Polytechnique Fédérale de Lausanne (EPFL), 1015 Lausanne, Switzerland
}

(Received 4 July 2017; published 22 February 2018)

\begin{abstract}
We explore the unconventional wave scattering properties of non-Hermitian systems in which amplification or damping are induced by time-periodic modulation. These non-Hermitian time-Floquet systems are capable of nonreciprocal operations in the frequency domain, which can be exploited to induce novel physical phenomena such as unidirectional wave amplification and perfect nonreciprocal response with zero or even negative insertion losses. This unique behavior is obtained by imparting a specific low-frequency time-periodic modulation to the complex coupling between lossless resonators, promoting only upward frequency conversion, and leading to nonreciprocal parametric gain. We provide a full-wave demonstration of our findings in a one-way microwave amplifier, and establish the potential of nonHermitian time-Floquet devices for insertion-loss free microwave isolation and unidirectional parametric amplification.
\end{abstract}

DOI: 10.1103/PhysRevLett.120.087401

In conventional media, wave scattering is usually reciprocal: the transmission coefficient from a source to a receiver remains the same if we interchange their locations or, in other words, the scattering matrix is always symmetric. This fundamental property of wave propagation is linked to microscopic reversibility, and holds for any linear time-invariant system in the absence of external time-odd bias $[1,2]$. There exist, however, many situations in which one would like to transmit waves unidirectionally by breaking reciprocity. For instance, nonreciprocal antennas that can emit and receive at the same frequency may allow for doubling the available bandwidth in the next generation of full-duplex telecommunication systems $[3,4]$.

In electromagnetic systems, the conventional way of breaking Lorentz reciprocity is the use of magnetic materials and an external magnetic field as time-odd external bias [5]. Magnets, however, are bulky, expensive, and to a large extent incompatible with integrated circuit technology and standard CMOS fabrication methods. For this reason, magnet-free nonreciprocal components have been developed based on nonlinearity [6-18] or time modulation [19-32]. Nonlinear systems strongly break reciprocity; however, they are fundamentally limited as isolators [33]. Time-Floquet systems represent a promising alternative to magnetic-free isolation, but they do not conserve frequency, and are thus associated with unavoidable insertion losses due to the energy lost in all the intermodulation frequency channels.

In a different field of research, non-Hermitian photonic systems that exploit the interplay between gain, loss, and the coupling between individual optical components have created a wealth of new opportunities in classical physics to generate and control the transmission of light [34-46]. While non-Hermitian systems by themselves cannot break reciprocity [47], they provide an interesting platform for nonunitary scattering, loss compensation, and wave amplification [39-46]. For instance, $\mathcal{P} \mathcal{T}$ symmetric systems can exhibit resonant localized amplification when operated in the broken phase, a phenomenon that can be exploited to obtain nonlinear responses at much lower power than in the exact phase [44], and induce low-threshold optical nonreciprocity $[45,46]$.

In this Letter, we investigate the unexplored physics of non-Hermitian systems for which non-Hermiticity is not due to the direct presence of material losses or gain, but is instead induced by periodic time modulation. We show that these non-Hermitian time-Floquet systems can be obtained by considering time-dependent coupling between lossless components, leading to a unique way to generate parametric loss or gain. Simultaneously, the breaking of time invariance in these systems can trigger nonreciprocal frequency conversions, allowing for highly efficient nonreciprocal amplification of the signal. By engineering at the same time non-Hermiticity and nonreciprocity, we overcome the insertion loss challenges inherent to Hermitian time-Floquet isolators, and obtain large nonreciprocal isolation with zero, or even negative insertion losses (signal amplification). We provide a full-wave demonstration of these findings at microwave frequencies.

Let us consider a simple time-invariant system described by the general two-by-two Hamiltonian $\mathbf{H}=\left[\omega_{1}, k_{12}\right.$; $\left.k_{21}, \omega_{2}\right]$. The usual route to induce non-Hermiticity of the system is to make $\omega_{1}$ and $\omega_{2}$ complex, which corresponds to adding gain and/or loss to the medium inside the resonators. However, imagine that instead, the coefficients $k_{12}$ and $k_{21}$ can depend on time: the Stokes principle of microscopic reversibility implies $k_{21}(t)=$ $k_{12}^{*}(-t)$, meaning that the system can potentially be 
non-Hermitian $\left(k_{21}(t) \neq k_{12}^{*}(t)\right)$ when $k_{12}(t)$ is not an even function of time. A particular example of such nonHermitian systems is obtained for $k_{12}(t)=K(t)=$ $k_{0}+\Delta k^{\prime} \cos (\Omega t)+j \Delta k^{\prime \prime} \sin (\Omega t)$, with $j^{2}=-1$, for which the relation $k_{21}(t)=k_{12}^{*}(-t)$ implies $k_{21}(t)=$ $k_{12}(t)=K(t)$, i.e., a non-Hermitian Hamiltonian with identical complex off-diagonal terms. In this Letter, we focus on the class of non-Hermitian two-level systems for which $\mathbf{H}=\left[\omega_{1}, K(t) ; K(t), \omega_{2}\right]$. Such Hamiltonians are not only non-Hermitian, but periodic in time with period $T=2 \pi / \Omega$, corresponding to time-Floquet non-Hermitian systems. Note that $k_{0}$ represents the static Hermitian part of the coupling, $\Delta k^{\prime} \cos (\Omega t)$ the time-dependent Hermitian part, and $j \Delta k^{\prime \prime} \sin (\Omega t)$ the time-dependent non-Hermitian part. In the following, we study the effect of each part of the coupling on the system dynamics and scattering properties.

An example of a device described by such a Hamiltonian is represented in Fig. 1. The two coupled resonators [coupling $K(t)$, resonance frequencies $\omega_{1}$ and $\omega_{2}$, $\left.\omega_{1}<\omega_{2}\right]$, are combined with two ports for incoming or outgoing wave signals, with decay rates $\gamma_{1}$ and $\gamma_{2}$. After applying the Floquet theorem [48] in time domain, we use standard temporal coupled mode theory (CMT) [49,50], writing the amplitudes $\alpha_{1}$ and $\alpha_{2}$ inside the two resonators as $\alpha_{1,2}(t)=\sum_{n} a_{1,2}^{n} e^{j(\omega+n \Omega) t}$, where $a_{1}^{n}$ and $a_{2}^{n}$ represent the time-independent complex amplitudes of the $n$th Floquet harmonic, and $\omega$ is the excitation frequency. Assuming excitation from port 1, the coupled-mode equations of the system are found as [51].

$$
\begin{aligned}
& \left(\omega+n \Omega-\omega_{1}-j \gamma_{1}\right) a_{1}^{n}-k_{0} a_{2}^{n}-\left(\frac{\Delta k^{\prime}+\Delta k^{\prime \prime}}{2}\right) a_{2}^{n-1} \\
& -\left(\frac{\Delta k^{\prime}-\Delta k^{\prime \prime}}{2}\right) a_{2}^{n+1}=\sqrt{2 \gamma_{1}} \delta_{n 0}, \\
& \left(\omega+n \Omega-\omega_{2}-j \gamma_{2}\right) a_{2}^{n}-k_{0} a_{1}^{n}-\left(\frac{\Delta k^{\prime}+\Delta k^{\prime \prime}}{2}\right) a_{1}^{n-1} \\
& \quad-\left(\frac{\Delta k^{\prime}-\Delta k^{\prime \prime}}{2}\right) a_{1}^{n+1}=0 .
\end{aligned}
$$

We see that $k_{0}$ couples identical frequencies, whereas the harmonic time-Floquet modulation couples each resonator with the two adjacent Floquet harmonics in the other resonator. For a Hermitian system $\left(\Delta k^{\prime \prime}=0\right)$, upward

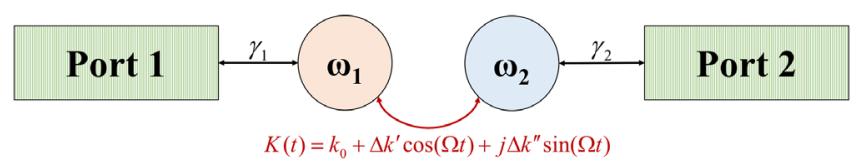

FIG. 1. Non-Hermitian time-Floquet system under study. Two coupled resonators with resonant frequencies $\omega_{1}$ and $\omega_{2}$ are connected to ports 1 and 2, with lifetimes $1 / \gamma_{1}$ and $1 / \gamma_{2}$. The complex internal coupling coefficient $K(t)$ is time dependent, with $K(t)=k_{0}+\Delta k^{\prime} \cos (\Omega t)+j \Delta k^{\prime \prime} \sin (\Omega t)$. and downward frequency conversion are equally efficient. Conversely, adding a non-Hermitian time-Floquet modulation $\left(\Delta k^{\prime \prime} \neq 0\right)$ allows one to tune the amount of energy that will undergo upward and downward frequency transitions. Crucially, the condition $\Delta k^{\prime}=\Delta k^{\prime \prime}$ is special as it enables only upward frequency conversion [51]. This unique capability is enabled by the non-Hermitian timeFloquet modulation, and totally impossible in previously considered Hermitian time-modulated systems [55-58].

To illustrate better the profound implication of this special condition on the scattering properties of the system, let us assume incidence from port 1 at $\omega=\omega_{1}$, and $\Omega=\omega_{2}-\omega_{1}$, so that the $n=+1$ harmonic is exactly at $\omega_{2}$ and will resonantly excite the second resonator. Figure 2 shows the norm of the field amplitudes $a_{1,2}^{n}$ of the $n=-1$, $n=0$, and $n=+1$ Floquet harmonics in various cases of interest. In the Hermitian case [Fig. 2(a), $\Delta k^{\prime \prime}=0$ ], the -1 , 0 , and +1 harmonics of the field amplitudes $\alpha_{1,2}$ all have significant energy. When we increase $\Delta k^{\prime}$, the energy of the -1 and +1 modes both increase, since all the frequency conversion rates are equal and proportional to $\Delta k^{\prime}$. Still for $\Delta k^{\prime \prime}=0$, another interesting phenomenon occurs if we force $k_{0}$ to become zero [Fig. 2(b)]: the system is driven to a condition where the second resonator cannot have a 0 harmonic, and transmission to the second port can only be at a frequency different than $\omega_{1}$. Yet, in both Hermitian cases of Figs. 2(a)-2(b), because upward and downward frequency conversion coefficients are equal, the transmission coefficient for incidence at port 2 with frequency $\omega_{2}$ to port 1 with frequency $\omega_{1}$ would be the same than the
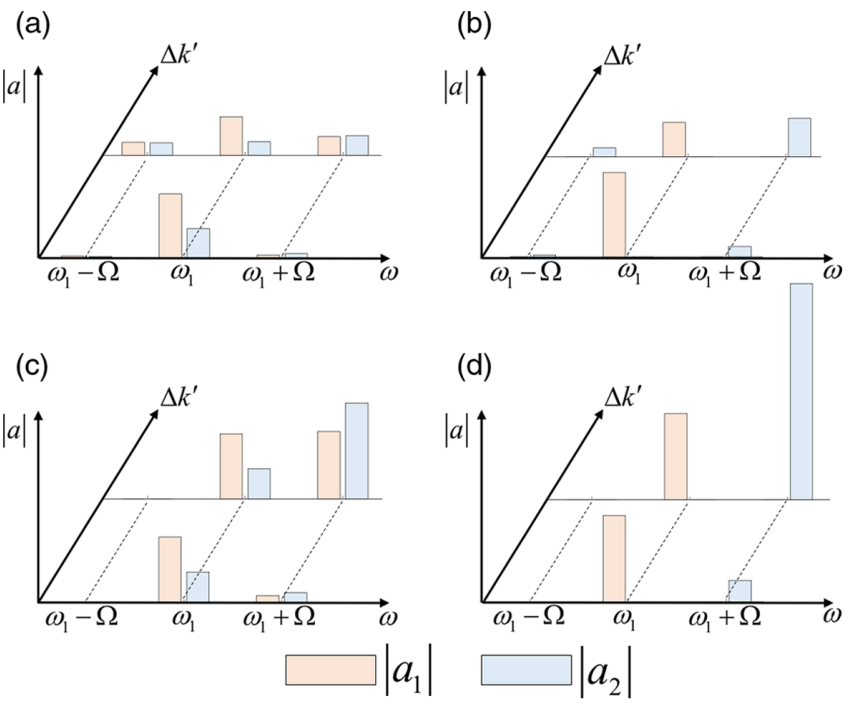

(d)

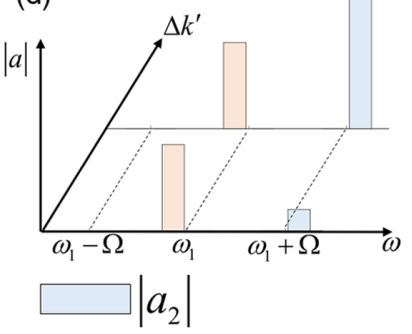

FIG. 2. Excited Floquet amplitudes for incidence at $\omega_{1}$ from port 1 . We represent the amplitudes of the three dominant Floquet harmonics in the first (orange) and second (blue) resonators for two different (arbitrary) values of $\Delta k^{\prime}$, and for (a) $\Delta k^{\prime \prime}=0$ and $k_{0} \neq 0$, (b) $\Delta k^{\prime \prime}=0$ and $k_{0}=0$, (c) $\Delta k^{\prime}=\Delta k^{\prime \prime}$ and $k_{0} \neq 0$, and (d) $\Delta k^{\prime}=\Delta k^{\prime \prime}$ and $k_{0}=0$. 
transmission coefficient for an input field at port 1 at $\omega_{1}$ to port 2 at $\omega_{2}$, and the system is reciprocal.

The situation is drastically different when we provide the system with a non-Hermitian time-periodic modulation [Figs. 2(c), 2(d)]. The plots are made under the special condition $\Delta k^{\prime}=\Delta k^{\prime \prime}$, which promotes absolute upward frequency conversion. As discussed above, this modulation never excites the -1 harmonic, whose amplitude is always identically zero, and forces the system to exhibit only the 0 and +1 Floquet harmonics [Fig. 2(c)]. In addition, if we force $k_{0}$ to become zero, the only remaining states are $a_{1}^{0}$ and $a_{2}^{+}$[Fig. 2(d)]. This means that the field is transmitted to port 2 only via $a_{2}^{+}$, through upward frequency conversion. Evidently, in these conditions a backward field incident on port 2 at $\omega_{2}$ will neither be downward converted to $\omega_{1}$ (since downward conversions are forbidden), nor be transmitted at $\omega_{2}$ through the zero harmonic (since $k_{0}=0$ ), and the transmission to port 1 will be identically zero. Therefore, the non-Hermitian time-Floquet system can exhibit perfect nonreciprocity: it transmits energy incident on port 1 to port 2, but has zero transmission for any signal incident on port 2. In Ref. [51], we provide a more complete study of the system's dynamics as $k_{0}$ and $\Delta k^{\prime \prime}$ change gradually, and demonstrate that this behavior remains true in a large vicinity of the condition $k_{0}=\Delta k^{\prime}-\Delta k^{\prime \prime}=0$.

Next, we move on to a quantitative study. Assuming time-harmonic incidence on port 1 , with $\psi_{\text {inc }}=\psi_{\text {inc }}^{0} e^{j \omega t}$, the transmitted field at the second port can be expanded as $\psi_{t}=\sum_{n} \psi_{t}^{n} e^{j(\omega+n \Omega) t}$, and we can define a transmission coefficient for each frequency harmonic as $S_{21}^{n}=\psi_{t}^{n} / \psi_{\mathrm{inc}}^{0}$. Here, only the $n=-1,0,1$ harmonics are important and we use the short-hand notations $S_{21}^{n=-1}=S_{21}^{-}, S_{21}^{n=0}=S_{21}^{0}$ and $S_{21}^{n=+1}=S_{21}^{+}$. Similarly, we define transmission coefficients for excitation from port 2 and note them $S_{12}^{-}, S_{12}^{0}$, and $S_{12}^{+}$. The definitions of scattering parameters are adequate since the system is linear [51]. Note that these quantities depend on the incident frequency, for instance, $S_{21}^{+}(\omega)$ corresponds to the field transmitted at port 2 at $\omega+\Omega$ when $\omega$ is sent at port 1 .

We represent in Fig. 3 the evolution of $\left|S_{21}^{+}\left(\omega_{1}\right)\right|$ and $\left|S_{12}^{-}\left(\omega_{2}\right)\right|$ as $\Delta k^{\prime}$ is gradually increased, comparing excitation from port 1 at $\omega_{1}$, and the reciprocal situation of excitation from port 2 at $\omega_{2}$. We also look at the transmission at the incident frequency $\left|S_{21}^{0}\left(\omega_{1}\right)\right|$ and $\left|S_{12}^{0}\left(\omega_{2}\right)\right|$. We consider two cases: (i) the static Hermitian case with $k_{0}=\Delta k^{\prime \prime}=0$ [Fig. 3(a)], and (ii) the non-Hermitian case with $k_{0}=0, \Delta k^{\prime}=\Delta k^{\prime \prime}$ [Fig. 3(b)]. Because $k_{0}=0$ in both cases, transmission at the excitation frequency is impossible, and $S_{21}^{0}$ and $S_{12}^{0}$ are always zero. In the Hermitian case, frequency conversion is symmetric and therefore the system is always reciprocal: we have $\left|S_{21}^{+}\left(\omega_{1}\right)\right|=\left|S_{12}^{-}\left(\omega_{2}\right)\right|$ regardless of $\Delta k^{\prime}$. Conversely, in the non-Hermitian case, transmission from port 2 to port 1 is identically zero regardless of the considered harmonic,

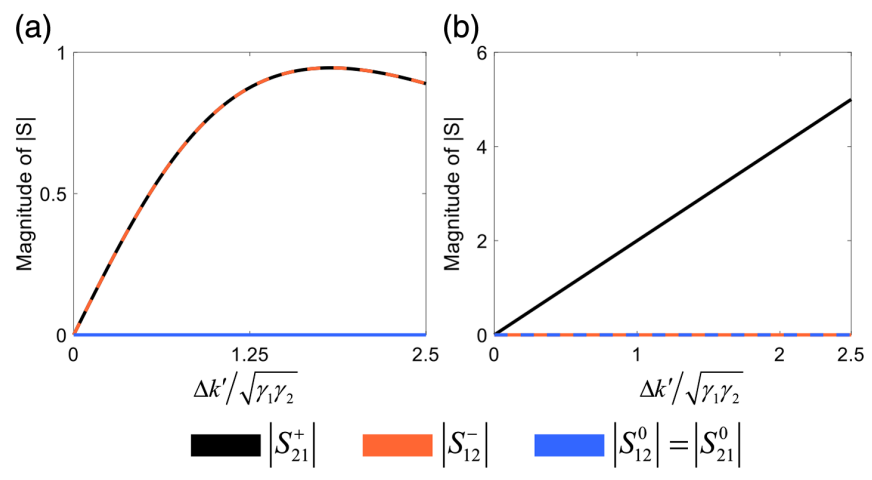

FIG. 3. Nonreciprocal gain in non-Hermitian time-Floquet systems. We plot the magnitude of the transmission coefficients to demonstrate the highly nonreciprocal behavior of the system. $S_{21}^{0}$ and $S_{21}^{+}$correspond to the transmissions to port 2, respectively, at $\omega_{1}$ and $\omega_{2}$, when a signal at $\omega_{1}$ is incident on port $1 . S_{12}^{0}$ and $S_{12}^{-}$correspond to the transmissions to port 1 , respectively, at $\omega_{2}$ and $\omega_{1}$, when a signal at $\omega_{2}$ is incident on port 2 . We compare the (reciprocal) Hermitian time-Floquet system with (a) $\Delta k^{\prime \prime}=0$ and $k_{0}=0$ to the (nonreciprocal) non-Hermitian time-Floquet system (b) $\Delta k^{\prime}=\Delta k^{\prime \prime}$ and $k_{0}=0$. Transmission through any other frequency channel is identically zero.

whereas $\left|S_{21}^{+}\left(\omega_{1}\right)\right|$ is nonzero, demonstrating the large nonreciprocal behavior. In addition, for sufficiently high values of $\Delta k^{\prime} / \sqrt{\gamma_{1} \gamma_{2}}$, we find that $\left|S_{21}^{+}\left(\omega_{1}\right)\right|$ can even reach values well above unity. Remarkably, the non-Hermitian time-Floquet modulation provides unidirectional parametric gain to the signal. This unique feature is a direct symptom of non-Hermiticity and is confirmed by direct finite-difference-time-domain (FDTD) simulations [51].

A closed-form analytical validation of this amplifying behavior can be obtained if we truncate the system of Eqs. (1), (2) to three Floquet harmonics. For a field incident at port 1 at frequency $\omega_{1}$, we find

$S_{21}^{+}=\frac{2 \sqrt{\gamma_{1} \gamma_{2}} \Delta k^{\prime}\left[\left(-\Omega-j \gamma_{2}\right)\left(\Omega-j \gamma_{1}\right)+k_{0}^{2}\right]}{\left[\left(-\Omega-j \gamma_{2}\right)\left(-j \gamma_{1}\right)-k_{0}^{2}\right]\left[\left(-j \gamma_{2}\right)\left(\Omega-j \gamma_{1}\right)-k_{0}^{2}\right]}$

If $k_{0}=0$, Eq. (3) becomes $S_{21}^{+}=-2 \Delta k^{\prime} / \sqrt{\gamma_{1} \gamma_{2}}$, which is identical with the results in Fig. 3(b), obtained numerically considering 201 Floquet harmonics. It is noteworthy that amplification can be obtained with arbitrarily small modulation depths $\Delta k^{\prime}=\Delta k^{\prime \prime}$ as long as the system is resonant enough, i.e., $\sqrt{\gamma_{1} \gamma_{2}}<\Delta k^{\prime}$. Using the same method, we have also checked that the transmission coefficients of the 0 Floquet harmonic $S_{21}^{0}$ and $S_{12}^{0}$ are proportional to $k_{0}$, and thus become zero when $k_{0}=0$, in perfect agreement with the results of Fig. 3.

We demonstrate our findings in a realistic full-wave device at microwave frequencies. The resonators are split rings with eigenfrequencies $f_{1}=3.1201$ and $f_{2}=$ $3.6921 \mathrm{GHz}$, the ports are microstrip transmission lines of width 0.333 and height $0.1778 \mathrm{~mm}$, and the substrate is 
FR4 with $\epsilon_{r}=4.5$. Between the resonators, we insert a capacitor of $C=0.15849 \mathrm{fF}$, which has a small modulation with depth $\Delta C=15.849 \mathrm{fF}$ and slow modulation frequency $f_{C}=572 \mathrm{MHz}$, in series with a resistor modulated in quadrature with $\Delta R=1 / \Delta C \omega$. We provide more details about the circuit implementation of these modulated elements in the Supplemental Material [51]. To minimize the static coupling strength $k_{0}$, we keep some distance between the components and place the resonators in antisymmetric positions (Fig. 4). The fields are computed using the three-dimensional finite-element method (FEM) method in frequency domain using a truncation of Maxwell's equations to three harmonics $\{-1,0,+1\}$.

Figure 4(a) shows the numerical results (solid lines), and compares them to the analytical CMT model (dashed lines). In Fig. 4(a), we plot the spectrum in $d B$ of the only nonzero transmission coefficients: $\left|S_{21}^{+}(\omega)\right|$, and $\left|S_{21}^{0}(\omega)\right|=\left|S_{12}^{0}(\omega)\right|$. Note that the $x$ axis of Fig. 4(a)

(a)
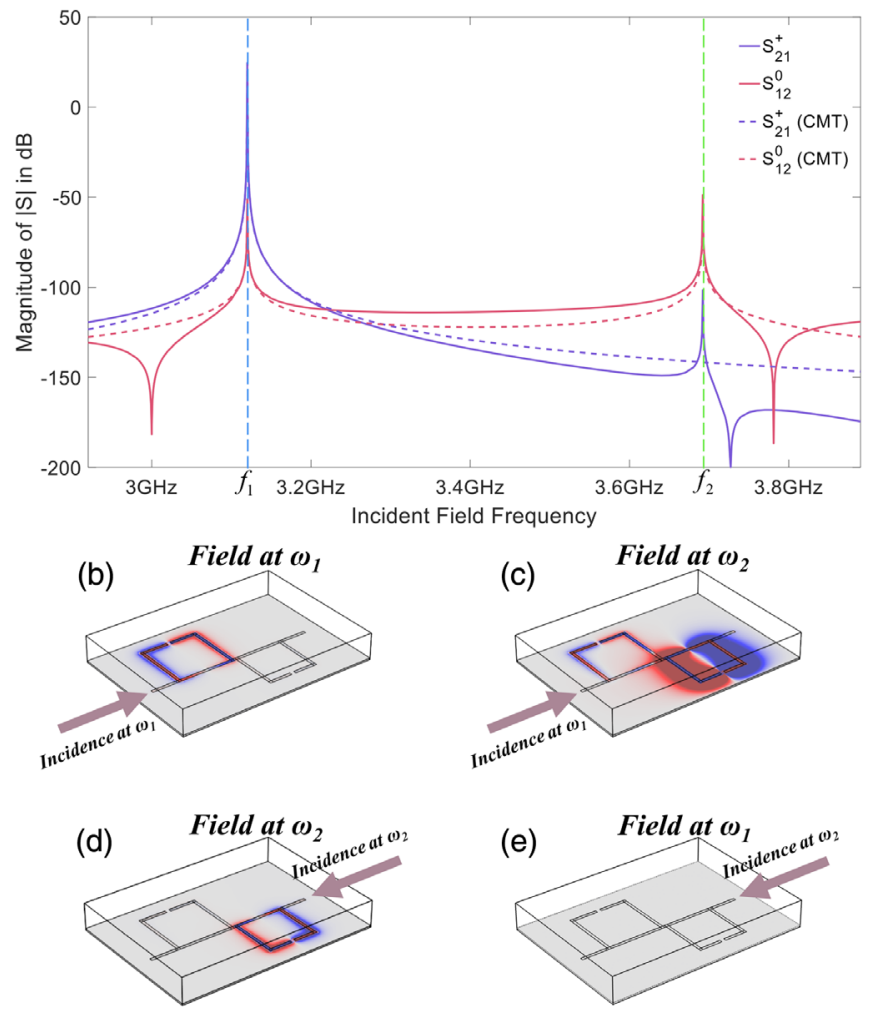

FIG. 4. Full-wave finite-element demonstration of non-reciprocal gain at microwave frequencies. The system is made of two microstrip ring resonators connected with a time-modulated capacitor in series with a time-modulated resistor [51]. (a) Spectrum of the scattering parameters demonstrating strong nonreciprocity and oneway amplification in a non-Hermitian time-Floquet system made of coupled split-ring resonators at $f_{1}$. Panels (b) and (c) correspond to incidence on port 1 at $\omega_{1}$ and show the vertical electric field component at $\omega_{1}$ and $\omega_{2}$, respectively. (d),(e) Same as (b) and (d) for incidence on port 2. All panels are plotted with the same scale, where blue correspond to a negative field and red to a positive field. corresponds to the frequency of the incident field. A point of abscissa $\omega$ on the curve $\left|S_{21}^{+}(\omega)\right|$, for instance, describes the amplitude of the Fourier component at $\omega+\Omega$ of the field transmitted to the second port. Therefore, the peak of $\left|S_{21}^{+}\right|$at the position $f_{1}$ of the $x$ axis corresponds to strong transmission to port two at $f_{1}+\Omega / 2 \pi=f_{2}$, i.e., transmission via upward frequency conversion. The level of this peak is above $30 \mathrm{~dB}$, meaning amplification. At any other frequency, including at $f_{2}$, the transmission from port 2 to port $1\left|S_{12}^{0}(\omega)\right|$ is always below $-50 \mathrm{~dB}$; i.e., $\left|S_{21}^{+}\left(f_{1}\right)\right|$ is more than $80 \mathrm{~dB}$ higher than $\left|S_{12}^{0}(\omega)\right|$ over the entire spectrum, which demonstrates that this parametric amplification phenomenon is indeed strongly nonreciprocal and well suited for signal isolation. The analytical model captures very well the physics involved, the only discrepancies being attributed to the inherent dispersion of the coupling coefficients, which is neglected in CMT. These discrepancies are extremely small, as they correspond to corrections that are $-100 \mathrm{~dB}$ below the incident field level. We find that the unidirectional transmission gain is linearly controlled by the modulation depth $\Delta C$ and the quality factor of the system, in perfect agreement with coupled mode theory. The simple Hamiltonian model is therefore a very good description of this system.

Below the spectrum, we show the distribution of the electric field (vertical component). Since this field is principally made up of two frequency components (at $\omega_{1}$ and $\left.\omega_{2}\right)$, we plot each component separately. When the signal is incident from port 1 at $\omega_{1}$ [Fig. 4(b)], the field component at $\omega_{1}$ remains isolated on the first resonator and no field is excited at frequency $\omega_{1}$ in the second resonator, preventing transmission to port 2 at this frequency. This is consistent with the very small static coupling $k_{0}$ between the rings. However, a nonzero field exists in the second resonator at frequency $\omega_{2}$ [Fig. 4(c)], which is consistent with upward frequency conversion. This field leaks out to port 2, giving nonzero transmission. When the signal is incident from port 2 at $\omega_{2}$, the field at $\omega_{2}$ remains localized on resonator 2 , and there is no significant transmission to port 1 [as shown in Fig. 4(d)]. Because of the upward-only frequency conversion property, downward frequency conversion is not allowed and the Floquet harmonic at $\omega_{1}$ is identically zero [Fig. 4(e)], making the system an extremely efficient isolator. The same phenomenon happens if $\omega_{1}>\omega_{2}$, providing $\Omega$ is negative; i.e., the helicity of the modulation is reversed to get downward frequency conversion. Therefore, by cascading two inverted systems, one with upward frequency conversion, and one with downward frequency conversion, we can make a nonreciprocal amplifier that operates without changing the frequency of the signal.

In conclusion, we have introduced a new class of physical systems in which a periodic drive induces not only nonreciprocity, but also non-Hermiticity. Different from previous works about time-Floquet non-Hermitian systems [59-62], which considered the time modulation 
of an already non-Hermitian static system, here nonHermiticity is induced by the modulation itself. These systems are capable of unidirectional parametric gain, and insertion-loss free isolation. Unidirectional amplification is of parametric nature, and obtained from a low modulation frequency, different from usual parametric amplification that requires pumping at the double frequency (this property, also found in QASERs [63], may be relevant in the design of efficient high frequency sources). A tunable microwave nonreciprocal amplifier may be readily implemented using voltage controlled capacitors and resistors combined with negative impedance converters [51], providing a way to solve the vexing insertion loss problem recurrent in conventional designs. Altogether, these exciting findings extend the reach of non-Hermitian physics to time-dependent systems and their applications.

This work was supported by the Swiss National Science Foundation (SNSF) under Grant No. 172487.

* To whom all correspondence should be addressed. romain.fleury@epfl.ch

[1] L. Onsager, Phys. Rev. 37, 405 (1931).

[2] H. B. G. Casimir, Rev. Mod. Phys. 17, 343 (1945).

[3] Y. Hadad, J. C. Soric, and A. Alù, Proc. Natl. Acad. Sci. U.S.A. 113, 3471 (2016).

[4] M. Jain, J. Il Choi, T. Kim, D. Bharadia, S. Seth, K. Srinivasan, P. Levis, S. Katti, and P. Sinha, Proceedings of the 17th Annual International Conference on Mobile Computing and Networking (ACM Press, New York, 2011), p. 301.

[5] L. J. Aplet and J. W. Carson, Appl. Opt. 3, 544 (1964).

[6] S. Tanaka, N. Shimomura, and K. Ohtake, Proc. IEEE 53, 260 (1965).

[7] K. Gallo, G. Assanto, K. R. Parameswaran, and M. M. Fejer, Appl. Phys. Lett. 79, 314 (2001).

[8] M. Soljačić, C. Luo, J. D. Joannopoulos, and S. Fan, Opt. Lett. 28, 637 (2003).

[9] S. Manipatruni, J. T. Robinson, and M. Lipson, Phys. Rev. Lett. 102, 213903 (2009).

[10] A. E. Miroshnichenko, E. Brasselet, and Y. S. Kivshar, Appl. Phys. Lett. 96, 063302 (2010).

[11] M. S. Kang, A. Butsch, and P. S. J. Russell, Nat. Photonics 5, 549 (2011).

[12] I. V. Shadrivov, V. A. Fedotov, D. A. Powell, Y. S. Kivshar, and N. I. Zheludev, New J. Phys. 13, 033025 (2011).

[13] V. Grigoriev and F. Biancalana, Opt. Lett. 36, 2131 (2011).

[14] T. Kodera, D. L. Sounas, and C. Caloz, Appl. Phys. Lett. 99, 031114 (2011).

[15] Z. Wang, Z. Wang, J. Wang, B. Zhang, J. Huangfu, J. D. Joannopoulos, M. Soljačić, and L. Ran, Proc. Natl. Acad. Sci. U.S.A. 109, 13194 (2012).

[16] L. Fan, J. Wang, L. T. Varghese, H. Shen, B. Niu, Y. Xuan, A. M. Weiner, and M. Qi, Science 335, 447 (2012).

[17] A. Kamal, A. Roy, J. Clarke, and M. H. Devoret, Phys. Rev. Lett. 113, 247003 (2014).

[18] A. Kamal and A. Metelmann, Phys. Rev. Applied 7, 034031 (2017).
[19] I. K. Hwang, S. H. Yun, and B. Y. Kim, Opt. Lett. 22, 507 (1997).

[20] S. Bhandare, S. K. Ibrahim, D. Sandel, Hongbin Zhang, F. Wust, and R. Noe, IEEE J. Sel. Top. Quantum Electron. 11, 417 (2005).

[21] Z. Yu and S. Fan, Nat. Photonics 3, 91 (2009).

[22] X. Huang and S. Fan, J. Lightwave Technol. 29, 2267 (2011).

[23] A. Kamal, J. Clarke, and M. H. Devoret, Nat. Phys. 7, 311 (2011).

[24] K. Fang, Z. Yu, and S. Fan, Phys. Rev. Lett. 108, 153901 (2012).

[25] M. Hafezi and P. Rabl, Opt. Express 20, 7672 (2012).

[26] H. Lira, Z. Yu, S. Fan, and M. Lipson, Phys. Rev. Lett. 109, 033901 (2012).

[27] C. G. Poulton, R. Pant, A. Byrnes, S. Fan, M. J. Steel, and B. J. Eggleton, Opt. Express 20, 21235 (2012).

[28] D. L. Sounas, C. Caloz, and A. Alù, Nat. Commun. 4, 2407 (2013).

[29] R. Fleury, A. Khanikaev, and A. Alù, Nat. Commun. 7, 11744 (2016).

[30] D.-W. Wang, H.-T. Zhou, M.-J. Guo, J.-X. Zhang, J. Evers, and S.-Y. Zhu, Phys. Rev. Lett. 110, 093901 (2013).

[31] N. A. Estep, D. L. Sounas, J. Soric, and A. Alù, Nat. Phys. 10, 923 (2014).

[32] N. Reiskarimian and H. Krishnaswamy, Nat. Commun. 7, 11217 (2016).

[33] Y. Shi, Z. Yu, and S. Fan, Nat. Photonics 9, 388 (2015).

[34] C. M. Bender and S. Boettcher, Phys. Rev. Lett. 80, 5243 (1998).

[35] K. G. Makris, R. El-Ganainy, D. N. Christodoulides, and Z. H. Musslimani, Phys. Rev. Lett. 100, 103904 (2008).

[36] S. Klaiman, U. Günther, and N. Moiseyev, Phys. Rev. Lett. 101, 080402 (2008).

[37] C. E. Rüter, K. G. Makris, R. El-Ganainy, D. N. Christodoulides, M. Segev, and D. Kip, Nat. Phys. 6, 192 (2010).

[38] A. Guo, G. J. Salamo, D. Duchesne, R. Morandotti, M. Volatier-Ravat, V. Aimez, G. A. Siviloglou, and D. N. Christodoulides, Phys. Rev. Lett. 103, 093902 (2009).

[39] Z. Lin, H. Ramezani, T. Eichelkraut, T. Kottos, H. Cao, and D. N. Christodoulides, Phys. Rev. Lett. 106, 213901 (2011).

[40] A. Regensburger, C. Bersch, M.-A. Miri, G. Onishchukov, D. N. Christodoulides, and U. Peschel, Nature (London) 488, 167 (2012).

[41] R. Fleury, D. Sounas, and A. Alù, Nat. Commun. 6, 5905 (2015).

[42] Y. D. Chong, L. Ge, and A. D. Stone, Phys. Rev. Lett. 106, 093902 (2011).

[43] P. Miao, Z. Zhang, J. Sun, W. Walasik, S. Longhi, N. M. Litchinitser, and L. Feng, Science 353, 464 (2016).

[44] H. Ramezani, T. Kottos, R. El-Ganainy, and D. N. Christodoulides, Phys. Rev. A 82, 043803 (2010).

[45] B. Peng, Ş. K. Özdemir, F. Lei, F. Monifi, M. Gianfreda, G. L. Long, S. Fan, F. Nori, C. M. Bender, and L. Yang, Nat. Phys. 10, 394 (2014).

[46] L. Chang, X. Jiang, S. Hua, C. Yang, J. Wen, L. Jiang, G. Li, G. Wang, and M. Xiao, Nat. Photonics 8, 524 (2014).

[47] D. Jalas, A. Petrov, M. Eich, W. Freude, S. Fan, Z. Yu, R. Baets, M. Popović, A. Melloni, J. D. Joannopoulos, 
M. Vanwolleghem, C. R. Doerr, and H. Renner, Nat. Photonics 7, 579 (2013).

[48] M. G. Floquet, Annales scientifiques de l'École Normale Supérieure 12, 47 (1883).

[49] H. A. Haus and W. Huang, Proc. IEEE 79, 1505 (1991).

[50] J. D. Joannopoulos, S. G. Johnson, J. N. Winn, and R. D. Meade, Photonic Crystals: Molding the Flow of Light, 2 nd ed. (Princeton University Press, Princeton, NJ, 2011).

[51] See Supplemental Material at http://link.aps.org/ supplemental/10.1103/PhysRevLett.120.087401 for a detailed analysis of the frequency conversion process, a discussion of the time-domain behavior, and a potential implementation of the coupling at microwaves, which includes Refs. [52-54].

[52] S. Assawaworrarit, X. Yu, and S. Fan, Nature (London) 546, 387 (2017).
[53] S. Hrabar, I. Krois, I. Bonic, and A. Kiricenko, Appl. Phys. Lett. 99, 254103 (2011).

[54] A. Antoniou, IEEE Trans. Circuit Theory 19, 209 (1972).

[55] L. Yuan, Y. Shi, and S. Fan, Opt. Lett. 41, 741 (2016).

[56] L. Yuan and S. Fan, Optica 3, 1014 (2016).

[57] T. Ozawa, H. M. Price, N. Goldman, O. Zilberberg, and I. Carusotto Phys. Rev. A 93, 043827 (2016).

[58] Y. Zhang and Y. Zhu, Phys. Rev. A 96, 013811 (2017).

[59] S. Longhi and G. Della Valle, Phys. Rev. A 87, 052116 (2013).

[60] S. Longhi, Europhys. Lett. 117, 10005 (2017).

[61] D. A. Telnov and S.-I. Chu, Phys. Rev. A 71, 013408 (2005).

[62] M. Chitsazi, H. Li, F. M. Ellis, and T. Kottos, Phys. Rev. Lett. 119, 093901 (2017).

[63] A. A. Svidzinsky, L. Yuan, and M. O. Scully, Phys. Rev. X 3, 041001 (2013). 